\title{
ALGEBRAIC STRATEGIES FOR PREDICTIONS IN ELEMENTARY MATHEMATICS EDUCATION
}

\author{
Gordana Nikolovska, Irena Stojkovska
}

\begin{abstract}
Algebraic thinking and reasoning is an important process in mathematics education, during which mathematical ideas are generalized from a specific set of instances, and the conclusion of the generalization is represented in different ways depending on the students' age. Algebraic strategies can be also used for practicing the students' prediction abilities, which can be beneficial while learning new concepts, and making connections to concepts that have been already learned. We have chosen patterns and number sequences as an initial step for exercising the prediction abilities in order to develop algebraic, functional and mathematical thinking among students in elementary education.
\end{abstract}

\section{INTRODUCTION}

Success in mathematics mostly depends on the development of sustainable interest among students about the subject. Students' interest in mathematics takes place when the student understand what the teacher talks, when problems or methods are interesting, when there is a possibility for independent reasoning and conclusions, when the student is convinced in his knowledge and cognitive abilities and when he discovers the usefulness and importance of this subject on real life examples. The introduction of mathematical concepts and connections between them requires teacher's correctness and consistency between the mathematical facts and the age of students, and respect of didactic principles, especially the principle of scientific approach, [2]. At the same time, it is extremely important to nurse the quality of mathematical thinking among students, because the final goal is not just to solve the problem, but to learn a method how to solve it. In fact, the heuristics in education can not be possible without the following qualities of the mathematical thinking being developed on a satisfactory level, there are: flexibility, depth, relevance, width and criticism, [1]. 
A particular challenge for teachers in elementary education is encouraging the functional thinking among students. In order to improve the conditions for practising the functional thinking, we emphasize the importance of practising the prediction abilities in mathematics education. Practising the students' prediction abilities is very important in order to maintain the curiosity and motivation for mathematics. The process of reasoning while predicting, leads to a special kind of learning. It is important that students ask themselves questions of the form "Why?" and "Why not?" and search for their answers. While predicting, students often use different types of problem visualizations, relate previously learned material with the problem they study, and reveal connections between mathematical objects and concepts, [7]. There are a small number of studies which put an emphasis on the predictions in mathematics education. Some of these studies are [3], [5], [7], [8].

In this paper, we consider the problem of practising the students' prediction abilities and introducing the functional thinking. We will consider several kinds of patterns and number sequences, together with the algebraic strategies for their treatment.

This paper is organized as follows. In Section 2 and Section 3, repeating patterns and growing patterns will be considered respectively. The number sequences will be discussed in Section 4, while the conclusions will be presented in Section 5.

\section{REPEATING PATTERNS}

Repeating patterns are one of more dominant type of patterns that students explore in their early years. They are used to describe the occurrence of the elements in the pattern by answering the questions "What is the next element in the sequence?" "What part is repeated?" or "What is missing?". In fact, recognizing how the elements are placed in these patterns, almost always leads to accurate predictions in answering the above questions, [9]. Here, we suggest several steps for investigating the repeating patterns. Each step contains questions or requests for students, which will help students to begin developing the functional thinking.

Example 1. Consider the repeating pattern shown in Figure 1. This is an example of a pattern that follows the rule AAB.

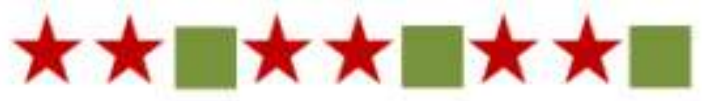

Figure 1. A repeating pattern 
The investigation of the pattern from Example 1 is through the following steps:

2-1. Copying the pattern in a notebook. By copying the elements of the pattern, the student will become familiar with its content and will sense the repetition of the figures (elements).

2-2. Continuing the pattern. The initial question in this step is "Which figure is next?". The answer includes predicting a figure to the right, but the pattern can also be continued to the left, by answering the question "Which figure precedes the first star?". The repetition of these questions, i.e. continuing the pattern with figures on the both sides, leads to the definition of the pattern rule.

2-3. Identification of the repeating elements (repeating set). The repeating set consists of the smallest number of elements that are repeated in the given order. In the repeating pattern from Example 1, it is the sequence "star, star, square" that is repeated. Students have to circle these groups (see Figure 2). There are more options and suggestions for choosing the repeating set (other correct answers are "star, square, star" and "square, star, star," if a series of figures that can be continued to the both sides is considered). But it is important to note that the simplest representation of a repeating set should be chosen, because this set continues to act like a pattern generator and should be suitable for analysis of the number of elements and the way they appear in the pattern. Therefore we say that the pattern in Figure 1 follows the rule AAB.

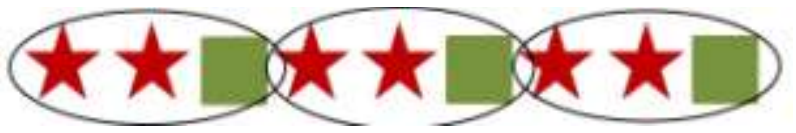

Figure 2. The repeating set in the pattern from Example 1

2-4. Questions, tables and generalizations. The definition of a repeating set is important for achieving the required generalization. To make sure that the students understand the creation of the pattern, and that they realize the power of their own predictions, here we propose several types of questions:

- Draw the pattern with 5 squares and 5 repeating sets. How many stars are there?

- If there are 10 repeating sets, how many squares and how many stars are there?

- If 12 figures of the pattern are drawn, so that the first figure is square, which is the twelfth figure?

Filling the Table 1 with the missing values, can be the same as asking the questions. Assume that the parts of repeating sets are not considered, but only whole repeating sets. 
Table 1. Table for the repeating pattern in Example 1

\begin{tabular}{|c|c|c|c|}
\hline $\begin{array}{c}\text { Number of } \\
\text { repeating sets }\end{array}$ & $\begin{array}{c}\text { Number } \\
\text { of stars }\end{array}$ & $\begin{array}{c}\text { Number } \\
\text { of squares }\end{array}$ & $\begin{array}{c}\text { Total number } \\
\text { of figures }\end{array}$ \\
\hline 20 & & & \\
\hline & 12 & & \\
\hline & & & 33 \\
\hline & & 40 & \\
\hline
\end{tabular}

Generalizing pattern for any arbitrary number of repeating sets is an interesting challenge for primary school students, and should not be excluded, though it might seem inappropriate for lower graders. Begin reasoning with a question as "How many squares will be there, if there are a total of $n$ repeating sets?". In this way, the relationship between the number of repeating sets, the total number of elements and the number of each kind of figure in the pattern is observed (the total number of squares is also $n$, the total number of stars is double i.e. $2 n$, and the total number of figures is three times greater i.e. $3 n$ ). Then, describing the rule of the pattern can be done with the following sentence: "Every third element is square, and the others are stars.", or the functional notation of the $i$-th element in the sequence will be:

$$
a(i)=\left\{\begin{array}{l}
\text { „star" }, \text { if } i \text { is not divisible by } 3 \\
\text { „square“, if } i \text { is divisible by } 3
\end{array}, i=1,2,3, \ldots\right.
$$

Note that, we decided to denote the $i$-th element of the sequence by $a(i)$, to get closer to the way of defining the function, as we try to give the functional dependence, and to be closer to algorithmic approach to problem solving that is used in programming.

2-5. Creating another pattern with the same rule and looking for real life applications. Using the methods of comparison and analogy for making conclusions is the cognitive level which confirms the understanding of the relation between the elements in the investigated pattern. Once it is established that the figures in the pattern follow some rule which is generated by the repeating set, it is clear that the nature of the elements is not important, but the rule in which they appear. We can start by changing colours, for example, colour the stars in yellow and the squares in blue. Then, we can replace the squares with circles and the stars with triangles etc. On the other hand, teachers have challenge and responsibility to reveal the application of the mathematical concepts which they teach in real life situations. Thus, they can ask students to give real life examples of repeating patterns, to identify the repeating sets and to describe the pattern rule. Such examples are the days of the week, the months of 
the year, then the tiles in front of the school building, the fence of the school yard, the buildings in our neighbourhood (some of them follow the "high-small" rule), and the musical rhythms.

\section{GROWING PATTERNS}

Patterns are powerful tool for developing the understanding of mathematical relationships and functions. With a good plan for treating the patterns, even the youngest students can be introduced to functional thinking, [4]. Growing patterns are also suitable for introducing students to the concept of linear function and its graph.

Example 2. Consider the growing pattern in Figure 3.

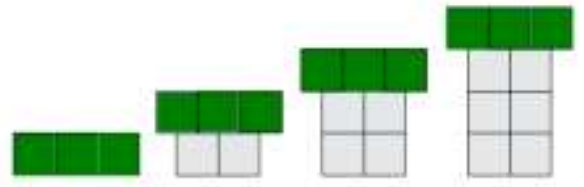

Figure 3. A growing pattern

The growing pattern in Example 2 will be examined in the following steps:

3-1. Copying the pattern in a notebook. The same step as with the repeating patterns, in order to make students to become familiar with its content and to make attempt to sense the rule.

3-2. Determining, numbering and comparing the steps. The growing nature of the pattern is obvious from the drawing, and the expectation of more squares in the next step is obvious. Note that the growing patterns are continued only on the right. So, the first question might be "What is the next step?". The following question might be "Which colour are the squares which number increases?". The first conclusion is that each step contains exactly three green squares. The number of grey squares "builds" the pattern, and there are no grey squares in the first step. The first step is called an initial step and the number 0 is assigned to it. So, we want to find and to describe the figure at the step 4 . The prediction is that this figure will have more grey squares then the figure at the step 3.

3-3. Counting and an initial prediction. The number of green and grey squares in each figure is counted. Then an initial prediction is made. Each figure contains exactly two grey squares more than the previous one, so we predict that the figure at step 4 has two grey squares more than the figure at step 3.

3-4. Filling a table. The first four columns in Table 2 are filled from the given part of the pattern and the remaining three columns are predictions. 
Table 2. Continuation of the growing pattern from Example 2

\begin{tabular}{|l|c|c|c|c|c|c|c|}
\hline Ordinal number of the step & 0 & 1 & 2 & 3 & $\mathbf{4}$ & $\mathbf{5}$ & $\mathbf{6}$ \\
\hline Number of blue squares & 3 & 3 & 3 & 3 & $\mathbf{3}$ & $\mathbf{3}$ & $\mathbf{3}$ \\
\hline Number of grey squares & 0 & 2 & 4 & 6 & $\mathbf{8}$ & $\mathbf{1 0}$ & $\mathbf{1 2}$ \\
\hline Total number of squares & 3 & 5 & 7 & 9 & $\mathbf{1 1}$ & $\mathbf{1 3}$ & $\mathbf{1 5}$ \\
\hline
\end{tabular}

3-5. Defining relationships, generalization and looking for real life applications. In growing patterns we look for the following two relationships: dependence between two consecutive steps - a recursive relation, and dependence between the number of the step and its structure - explicit or functional expression, [4]. A recursive relation between two consecutive steps is found right after the counting. It is expected that the students from the lower classes will describe the pattern as $3+2+2+2+\ldots$ pattern. The little older students should consider the relationship between the number of grey squares and the number of the step i.e. this relationship can be described by $0 \rightarrow 0,1 \rightarrow$ $2,2 \rightarrow 4,3 \rightarrow 6$, etc. and they should recognize that the number of grey squares is twice more then the number of step. Finally, if we add the constant number of green squares, we will have: $0 \rightarrow 3+0 \cdot 2,1 \rightarrow 3+1 \cdot 2,2 \rightarrow 3+2 \cdot 2,3 \rightarrow 3$ $+3 \cdot 2$, etc. Now, if we denote by $i$ the number of step, the pattern in Example 2 can be described by $3+2 \cdot i$ pattern, where $i$ is any natural number or zero, i.e. the total number of squares in the $i$-th step can be defined by

$$
a(i)=3+2 \cdot i, i=0,1,2,3, \ldots
$$

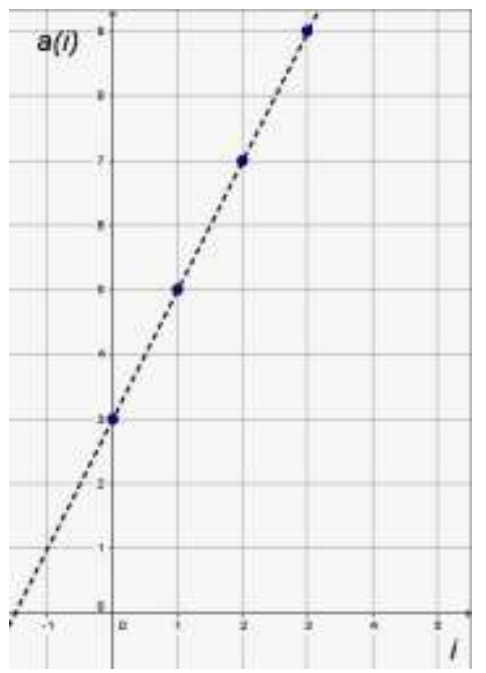

Figure 4. A graphical representation of the growing pattern from Example 2.

For students from higher classes, the growing linear patterns can be a good introduction to the graph of linear functions. If the number of step denoted by $i$ 
is read from $x$-axis, and the total number of squares denoted by $a(i)$ is read from the $y$-axis, then the growing pattern in Example 2 can be presented graphically as in Figure 4. This graph can be also used for predictions.

To understand better the concept of growing patterns, it is good to look for real life examples. Some of them are: the age of a person (in years or months, observed at regular intervals), the construction phases of a rocket (first the head is built, then the modules are added one by one from below), train with a locomotive and wagons that are added at each station in equal portions or slightly more complex growing patterns such as the number of snowflake rays at each stage of its formation, the yearly number of tree branches etc.

\section{NUMBER SEQUENCES}

When counting the growing patterns, a number sequence is formed such that for each step of the pattern corresponds an element from the sequence. We will explore linear, double rule and quadratic dependence in number sequences.

\subsection{Linear dependence}

Let us consider the following example:

Example 3. Given the sequence 1, 4, 7, 10, 13, 16, .. Find the rule and continue the sequence with at least three elements.

Table 3. Prediction of the elements of the sequence from Example 3 by calculating the differences of two consecutive elements

\begin{tabular}{|c|c|c|}
\hline $\begin{array}{c}\text { Ordinal number } \\
\text { of the element } i\end{array}$ & $\begin{array}{c}\text { Value of the } \\
\text { element } a(i)\end{array}$ & $\begin{array}{c}\text { Difference of two consecutive } \\
\text { Elements } a(i+1)-a(i)\end{array}$ \\
\hline 1 & 1 & 3 \\
\hline 2 & 4 & 3 \\
\hline 3 & 7 & 3 \\
\hline 4 & 10 & 3 \\
\hline 5 & 13 & $\mathbf{3}$ \\
\hline 6 & 16 & $\mathbf{3}$ \\
\hline $\mathbf{7}$ & $\mathbf{1 9}$ & $\mathbf{3}$ \\
\hline $\mathbf{8}$ & $\mathbf{2 2}$ & \\
\hline $\mathbf{9}$ & $\mathbf{2 5}$ & $\mathbf{2}$ \\
\hline
\end{tabular}


We will investigate the sequence in the following way: denote by $i$ the ordinal number of an element in the sequence, and by $a(i)$ its value. Then, fill in the Table 3 the first six given numbers in the sequence. Calculate the differences of any two consecutive elements. It can be easily realised that at each step this difference is equal to 3 . Then, the students can make a prediction for the next three steps. In fact, we predict that in the next three steps the difference of any two consecutive elements will be 3, so we write down these values in the third column, and then calculate the unknown elements $a(7), a(8)$ and $a(9)$ by knowing the preceding elements. So, the sequence will be continued by the following three elements 19, 22 and 25 .

It is important to note that in this way we approach a step closer to the functional dependence, which is one of our the goals in this investication of patterns and number sequences. Namely, from $a(i+1)-a(i)=3, i=1,2,3, \ldots$ we have

$$
a(i+1)=a(i)+3, i=1,2,3, \ldots
$$

i.e. the rule of this sequence is that "the next number is 3 more than the previous one". Considering the condition $a(1)=1$, the relation (3) becomes a recursive way of defining the sequence from Example 3.

Then, by a similar reasoning as in Example 2, observing the elements of the sequence we can see that each element contains the number 3 as many times as its ordinal number decreased by 1 and the element is this value increased by 1 , which can be written as

$$
a(i)=3(i-1)+1=3 i-2, i=1,2,3, \ldots
$$

We can also make the corresponding graphical representation of the sequence.

\subsection{Number sequences with a "double" rule}

In Example 3, the linear dependence refers to the relation between the values of the elements and its place in the sequence. The linear dependence can be more complicated, as we will show on the following example.

Example 4. Let us consider the sequence $0,-1,2,-2,4,-3,6, \ldots$ Find the rule and continue the sequence with at least three elements.

If we start writing the elements of the sequence in a table like the one for the previous Example 3, we will immediately run into difficulties, namely, the rule will not be so obvious. Therefore, we will approach this problem graphically, which will help us to predict the next elements (see Figure 5). From the graph of the sequence we can see that the elements of the sequence that are in odd places, 
they are $a(1)=0, a(3)=2, a(5)=4, a(7)=6$, follow a linear dependence, and the same is with the elements of the sequence that are in even places, $a(2)=-1, a(4)=-2, a(6)=-3, \ldots$ The lines that describe these dependences are $y=x-1$, for the elements on odd places and $y=-x / 2$, for the elements on even places. So, the "double" rule of the sequence from Example 4 is

$$
a(i)=\left\{\begin{array}{ll}
i-1, & \text { if } i \text { is odd } \\
-i / 2, & \text { if } i \text { is even }
\end{array} \quad i=1,2,3, \ldots\right.
$$

According to this rule and by the graphical representation of the sequence, we get the next three elements of the sequence: $-4,8$ and -5 .

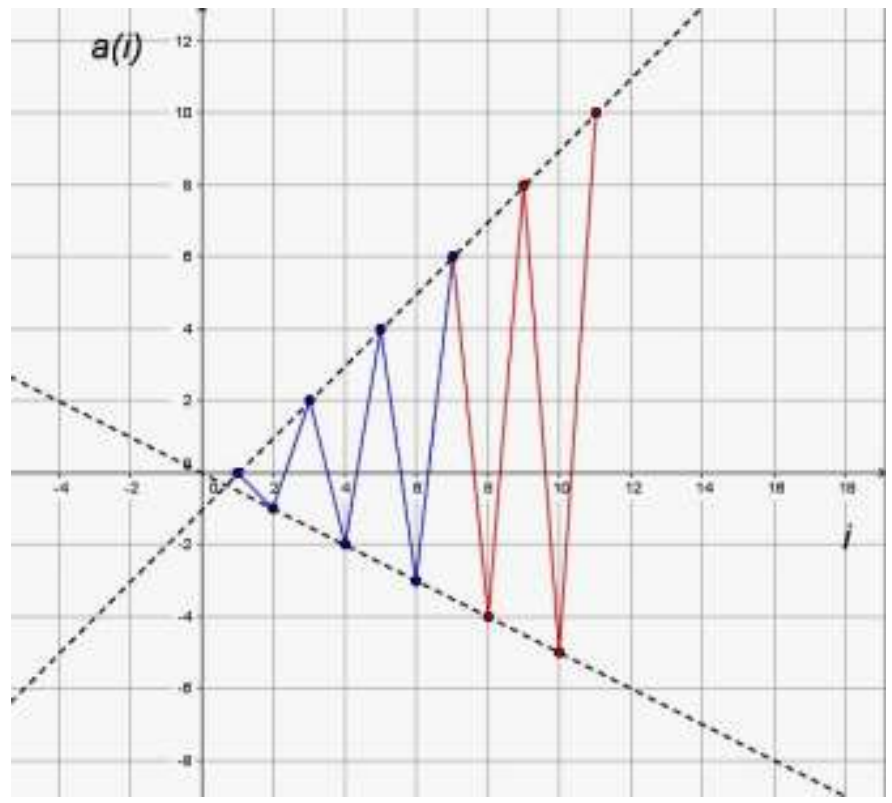

Figure 5. A graphical representation of the sequence with a "double" rule from Example 4

We can also derive the same result, without looking the graphical representation of the whole sequence, but analyzing the differences of two consecutive elements in the both subsequences (odd and even places subsequences respectively) as it is done in Table 3 for Example 3.

\subsection{Quadratic dependence}

The method of prediction by using a table with the differences, as in Example 3, can be used for investigating the polynomial dependence between the elements of the sequence and their place in the sequence. A justification lies 
in the First Newton's interpolating polynomial, [6]. Here, we will focus on the quadratic dependence, so we have to calculate the differences between two successive elements (the first order differences) and the differences between two successive differences of the first order (the second order differences). We will illustrate the prediction method on the following example.

Example 5. A triangle has no diagonals, a quadrilateral has two, a pentagon has five and a hexagon has nine diagonals (Figure 6). How many diagonals have a heptagon?
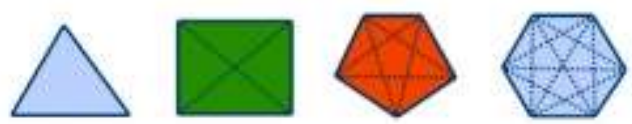

Figure 6. Polygons and their diagonals

We will approach the problem by forming a sequence of total number of diagonals, and we should predict how to continue it for one step. The following questions will help the students to complete this task: How does the sequence whose members are the total number of diagonals in a polygon look like? How does the corresponding sequence of total numbers of sides of the corresponding polygon look like? How does the total number of diagonals increase, each time the number of sides increases by 1 ? What is the dependence between the diagonals and the total number of sides?

After the discussion, students will realize that the problem is reduced to finding the fifth element of the sequence $0,2,5,9, \ldots$, while the "ordinal number" of the first element of this sequence is 3 , because the first polygon is a triangle. So, analysing the four polygons, the Table 4 is filled with their number of sides $n$, the corresponding number of diagonals $a(n)$, the first order differences $b(n)=a(n+1)-a(n)$ and the second order differences $b(n+1)-b(n)$.

Table 4. Investigating the sequence of total number of diagonals in polygons from Example 5

\begin{tabular}{|c|c|c|c|}
\hline $\begin{array}{c}\text { Number of } \\
\text { sides } \\
n\end{array}$ & $\begin{array}{c}\text { Number of } \\
\text { diagonals } \\
a(n)\end{array}$ & $\begin{array}{c}\text { First order } \\
\text { differences } \\
b(n):=a(n+1)-a(n)\end{array}$ & $\begin{array}{c}\text { Second order } \\
\text { differences } \\
b(n+1)-b(n)\end{array}$ \\
\hline 3 & 0 & 2 & 1 \\
\hline 4 & 2 & 3 & 1 \\
\hline 5 & 5 & 4 & $\mathbf{1}$ \\
\hline 6 & 9 & $\mathbf{5}$ & \\
\hline 7 & $\mathbf{1 4}$ & & \\
\hline
\end{tabular}


From the Table 4, we can see that the second order differences are equal to 1, so we predict that the next unknown second order difference is also equal to 1 , from where it follows that the next first order difference is 1 more than 4 , so it is equal to 5, and finally, the prediction for the number of diagonals of a heptagon is $9+5=14$ diagonals.

We also want to derive a functional dependence of the total number of diagonals and the number of sides of a polygon. So, first we predict that the second order differences are equal to 1 i.e. $b(n+1)-b(n)=1$, for all $n=3,4, \ldots$, from where, using the definition $b(n):=a(n+1)-a(n)$, we get

$$
a(n+2)=2 a(n+1)-a(n)+1, n=3,4, \ldots,
$$

which is a recursive definition of a sequence of total number of diagonals in a polygon.

A different recursive definition can be obtained by realising and predicting that $b(n)=n-1, \quad n=3,4, \ldots . \quad$ Substituting again in the definition $b(n):=a(n+1)-a(n)$, we get

$$
a(n+1)=a(n)+n-1, n=3,4, \ldots
$$

To find the functional dependence of the total number of diagonals and the number of sides in a polygon, we start with $a(3)=0$, and using the recursive formula (7) we get the following representations

$$
\begin{aligned}
& a(4)=a(3)+3-1=0+3-1, \\
& a(5)=a(4)+4-1=0+3-1+4-1, \\
& a(6)=a(5)+5-1=0+3-1+4-1+5-1, \\
& a(7)=a(6)+6-1=0+3-1+4-1+5-1+6-1,
\end{aligned}
$$

and so on, which help us to make the following generalization (which can be also viewed as a prediction):

$$
\begin{aligned}
a(n) & =(3+4+5+6+\ldots+n-1)-(n-3)= \\
& =(1+2+3+4+5+6+\ldots+n-1)-1-2-(n-3)= \\
& =\frac{(n-1) n}{2}-n=n\left(\frac{n-1}{2}-1\right)=\frac{n(n-3)}{2},
\end{aligned}
$$

$n=3,4, \ldots$. In this derivation, the knowledge of the formula for the sum of the first $n$ positive integers is needed.

\section{CONCLuSiOnS}

Investigation of the patterns and number sequences can contribute a lot in practising prediction skills. However, the prediction (the shape of the next 
figure in the pattern, i.e. the value of the next element in the sequence) should not be the only objective of this kind of problems. Looking for the functional dependence of the figures in the pattern or the elements of the sequence is a step forward in the investigation and in the practice of prediction skills. This practice will help students to connect already learned material to the new material, and students can become more confident and motivated by knowing that they come to the new knowledge by themselves. Also, the investigation of patterns and number sequences offers opportunities to develop mathematical talks between the teacher and students, and among the students. So, this kind of problems should find more often its way in math classes. They are suitable for "worm-up" activities at the beginning of a class.

\section{COMPETING INTERESTS}

Authors have declared that no competing interests exist.

References

[1] Р. Малчески, Методика на наставата по математиката, Скопје, 2016

[2] Н. Целакоски, Дидактика на математиката, Скопје, 1993

[3] M. Battista, Fifth graders' enumeration of cubes in 3D arrays: Conceptual progress in an inquiry-based classroom, Journal for Research in Mathematics Education, 30 (1999), 417-448.

[4] R. Beatty, Exploring the Power of Growing Patterns, What Works?: Research into Practice, Maniscript \#55, Student Achievement Division and Ontario Association of Deans of Education, August 2014

[5] G. Buendía, F. Cordero, Prediction and the periodical aspect as generators of knowledge in a social practice framework, Educational Studies in Mathematics, 58 (2005), 299-333.

[6] R. L. Burden, J. D. Faires, Numerical Analysis, 9th Ed., Brooks/Cole, Boston, MA, 2011

[7] L. A. Kasmer, O. K. Kim, The nature of student predictions and learning opportunities in middle school algebra, Educational Studies in Mathematics 79(2) (2012), 175-191

[8] O. K. Kim, L. Kasmer, Effect of using prediction questions in middle school algebra classrooms, Proceedings of the 9th International Conference "Mathematics Education in a Global Community" (2007), $359-363$ 
[9] E. Warren, T. Cooper, Using repeating patterns to explore functional thinking, APMC 11(1) (2006), 9-14

Department of Mathematics, Faculty of Natural Sciences and Mathematics, Ss. Cyril and Methodius University, Skopje, R. Macedonia

E-mail address: gordana.nikolovska.93@gmail.com

Department of Mathematics, Faculty of Natural Sciences and Mathematics, Ss. Cyril and Methodius University, Skopje, R. Macedonia

E-mail address: irenatra@pmf.ukim.mk 
